\title{
Efficacy of fixed-dosing aflibercept for treating polypoidal choroidal vasculopathy: 1-year results of the VAULT study
}

\author{
Joo Eun Lee ${ }^{1}$ - Jae Pil Shin ${ }^{2}$ • Hyun Woong Kim ${ }^{3}$ - Woohyok Chang ${ }^{4,5} \cdot$ Yu Cheol Kim ${ }^{6}$. \\ Sang Joon Lee ${ }^{7} \cdot$ In Young Chung ${ }^{8} \cdot$ Ji Eun Lee ${ }^{9,10,11} \cdot$ VAULT study group
}

Received: 16 June 2016 /Revised: 18 August 2016 / Accepted: 25 August 2016 /Published online: 14 September 2016

(C) Springer-Verlag Berlin Heidelberg 2016

\begin{abstract}
Purpose To investigate fixed-dosing aflibercept for treating polypoidal choroidal vasculopathy (PCV).

Methods This phase IV, prospective, single-arm, interventional case series was conducted in eight centers. Forty treatment-naïve PCV patients were administered three monthly doses of intravitreal aflibercept $(2.0 \mathrm{mg})$ and an injection every 2 months thereafter. Best-corrected visual acuity (BCVA) and central subfield macular thickness (CSMT) were measured at each visit. Fluorescein and indocyanine green angiography (ICGA) were performed at baseline, 3 and 12 months. The primary outcome measure was the proportion of patients who maintained BCVA $(<15$ letters loss) at 12 months. Changes in BCVA, macular appearance, and polypoidal lesion appearance were also examined.
\end{abstract}

ClinicalTrials.gov number, NCT01950741

The authors have full control of all primary data, and agree to allow Graefe's Archive for Clinical and Experimental Ophthalmology to review our data upon request.

Ji Eun Lee

jlee@pusan.ac.kr

1 Department of Ophthalmology, Haeundae Paik Hospital, Inje University College of Medicine, Busan, South Korea

2 Department of Ophthalmology, Kyungpook National University Hospital, Deagu, South Korea

3 Department of Ophthalmology, Busan Paik Hospital, Inje University College of Medicine, Busan, South Korea

4 Department of Ophthalmology, Yeungnam University College of Medicine, Daegu, South Korea
Results Thirty-five eyes $(87.5 \%)$ had maintained BCVA at 12 months. Average BCVA was significantly higher at 12 months (20/53, 64.2 letters) than at baseline (20/80, 55.1 letters, 9-letter gain; $P<.001)$. Mean CSMT was significantly lower at 12 months $(253.6 \mu \mathrm{m})$ than at baseline $(365.2 \mu \mathrm{m}$, $P<.001)$. The macula was dry in $32(76.2 \%), 27(64.3 \%)$, and 24 eyes $(60.0 \%)$ at 3,6 , and 12 months respectively. Fourteen eyes $(33.3 \%)$ had a fluid recurrence or increase at 6 months, and they had a significantly lower vision gain $(P=.005)$ than other patients at 12 months. Complete polyp regression occurred in 26 eyes (66.7\%) at 12 months.

Conclusions Fixed-dosing aflibercept showed favorable outcomes in PCV patients at 12 months. However, some patients had worse outcomes because of fluid recurrence during maintenance dosing, and these patients would require additional treatments.

5 Chang's Retina Center, Daegu, South Korea

6 Department of Ophthalmology, Dongsan Medical Center, Keimyung University School of Medicine, Daegu, South Korea

7 Department of Ophthalmology, College of Medicine and Institute of Medicine, Kosin University, Busan, South Korea

8 Department of Ophthalmology, Gyeongsang National University School of Medicine, Jinju, South Korea

9 Department of Ophthalmology, Pusan National University Hospital, \#179 Gudeok-ro, Seo-gu, Busan 602-739, South Korea

10 Department of Ophthalmology, College of Medicine, Pusan National University, Yangsan, South Korea

11 Biomedical Research Institute, Pusan National University Hospital, \#179 Gudeok-ro, Seo-gu, Busan 602-739, South Korea 
Keywords Aflibercept - Age-related macular degeneration . Polypoidal choroidal vasculopathy $\cdot$ Fixed regimen

\section{Introduction}

Polypoidal choroidal vasculopathy (PCV) is a subtype of exudative age-related macular degeneration (AMD) that can cause permanent vision loss due to hemorrhage, exudation, macular edema, and disciform scar formation. This type of AMD has been reported to account for approximately $10 \%$ of exudative AMD cases in Western populations [1] and approximately $50 \%$ of exudative AMD cases in Asian populations [2]. Ranibizumab is now the first-line therapy for treating $\mathrm{PCV}$, in replacement of photodynamic therapy (PDT), because it significantly improves vision after monthly intravitreal injections of the agent [3, 4].

Interestingly, $\mathrm{PCV}$ responds differently than typical AMD to intravitreal anti-vascular endothelial growth factor (VEGF) treatments. Persistent subretinal fluid [5] and polypoidal vessels $[6,7]$ are common despite bevacizumab or ranibizumab treatment. However, PDT with verteporfin was superior to ranibizumab monotherapy over the short term in terms of anatomical parameters [8]. Nevertheless, anti-VEGF treatment seems more promising than PDT. Visual acuity was not maintained with PDT treatment over the long term [9, $10]$ and eyes treated with ranibizumab had better functional outcomes than eyes treated with PDT after 1 year $[11,12]$. Additionally, the VEGF Trap-Eye Investigation of Efficacy and Safety in Wet AMD (VIEW) 1 and 2 studies demonstrated that aflibercept is equally effective for treating wet AMD as ranibizumab, but it has a longer treatment interval [13]. Several studies have also shown that treatment of PCV with aflibercept results in good functional results and, frequently, polyp closure [14-17].

However, prior studies were limited by a short follow-up period [14, 15], a single-center setting [16], or a retrospective design [17]. Although the VIEW studies included PCV patients, the diagnosis was not confirmed with indocyanine green angiography (ICGA). The purpose of the current study was to prospectively evaluate the effect of intravitreal injection of VEGF-Trap Eye on polypoidal choroidal vasculopathy in treatment-naïve patients (VAULT).

\section{Methods}

\section{Study design}

The VAULT study was designed as a phase IV, prospective, multicenter, interventional, single-arm, single-dose clinical trial (ClinicalTrials.gov ID NCT01950741). Patients diagnosed with PCV were recruited between September 2013 and
July 2014 at eight hospitals across South Korea. The protocol was approved by the Institutional Review Board/Ethics Committee at each participating hospital. All study conduct adhered to the tenets of the Declaration of Helsinki, and all subjects provided written informed consent to participate in this study.

\section{Inclusion and exclusion criteria}

A scanning laser ophthalmoscope (HRA-2; Heidelberg Engineering, Heidelberg, Germany) was used to perform fluorescein angiography (FA) and indocyanine green angiography (ICGA). A PCV diagnosis was given if ICGA showed polypoidal vessel dilation with or without a branching vascular network (BVN). Subjects were considered for participation if all of the following were true: diagnosed with the PCV subtype of exudative AMD via ICGA, polypoidal lesion located within $3,000 \mu \mathrm{m}$ of the foveal center, and best-corrected visual acuity (BCVA) between 20/40 and 20/320. Subjects also had to have fluorescein dye leakage (as shown on FA) and/or evidence of intraretinal, subretinal, or sub-retinal pigment epithelium (RPE) fluid (as shown on optical coherence tomography $[\mathrm{OCT}])$.

Subjects were excluded if any of the following were true: 1) presence of a subretinal hemorrhage or other pathology that blocked at least $50 \%$ of the PCV lesion in ICGA, 2) history of intravitreal anti-VEGF or photodynamic therapy, 3) history of intraocular surgery (except uncomplicated cataract surgery performed more than 3 months before enrollment), 4) other ocular disease known to affect vision (e.g., glaucoma, visually significant cataract), 5) uncontrolled systemic disease (e.g., diabetes, high blood pressure, cardiovascular disease, cerebrovascular disease), 6) active infection inside or around the eye, or 7) hypersensitive reaction to aflibercept or other injection components.

\section{Treatment and assessment schedule}

Intravitreal injection of aflibercept $(2.0 \mathrm{mg}$ in $0.05 \mathrm{ml})$ through the pars plana was performed under aseptic conditions. Three monthly doses were followed by a maintenance injection every 2 months for a total of a 12-month treatment period (total of seven injections). At each visit, BCVA (ETDRS chart) and intraocular pressure were measured. Slit-lamp biomicroscopy, color fundus photography, and spectral-domain OCT were performed before injections. Using OCT images, central subfield macular thickness was measured as the average thickness of the central $1 \mathrm{~mm}$ thickness map measurement area. Both FA and ICGA were obtained at baseline, 3 and 12 months. Photodynamic therapy with verteporfin was used as a rescue treatment, and was performed at the treating physician's discretion at 6 months or later. 


\section{Outcome measures}

The primary outcome measure was the proportion of patients who maintained BCVA, defined as losing $<15$ ETDRS letters over the 12-month study period. Secondary outcome measures included the following: mean change in BCVA from baseline at 12 months, proportion of patients with a BCVA of $20 / 200$ or better at 12 months, proportion of patients with a BCVA of 20/40 or better at 12 months, proportion of patients who had complete intraretinal/subretinal fluid resolution at 12 months, proportion of patients who had complete polypoidal lesion disappearance at 12 months based on ICGA.

All study images were collected from each participating center, and were interpreted by three of the authors (JEL, JEL, and HWK). When disagreements occurred, images were discussed until a consensus was reached. Polyp changes were categorized at 3 and 12 months as complete regression, partial regression, persistent, or newly developed. Enlargement of BVNs was also assessed. Macular drying was categorized as complete, incomplete, or persistent, and fluid levels were compared with those at the previous visit. The BVN area and polypoidal lesion size were measured in ICGA images at baseline using ImageJ software (National Institutes of Health, ver. 1.49). The size of the largest polypoidal lesion was defined as the largest linear dimension of the largest polypoidal dilation in ICGA images. Location of the PCV lesion and the polypoidal lesion closest to the foveal center was categorized as subfoveal, juxtafoveal, and extrafoveal.

\section{Statistical analyses}

Non-parametric tests (Wilcoxon signed-rank and MannWhitney U tests) were used to compare measured values, and Spearman's ranked correlation was used to examine correlations between measurements. Categorical variables were analyzed using Fisher's exact test or Chi-square test. Statistical significance was defined as $P<.05$. SPSS statistical software (ver. 17.0 for Windows, IBM Corp., Armonk, NY, USA) was used to perform statistical analyses.

\section{Results}

A total of 48 patients were enrolled in the study, but 40 patients (27 male, 13 female) were ultimately included in analyses (Table 1). Three patients dropped out of the study before the end of the 12-month follow-up period, and five patients were excluded because ICGA did not show definitive PCV. The mean age of included subjects was $67.0 \pm 9.2$ years (range: 44-84 years). Location of vascular lesion was subfoveal in 19 eyes. Mean BVN area was $3.39 \pm 4.10 \mathrm{~mm}^{2}$ (range: $0.32-24.81 \mathrm{~mm}^{2}$ ). Number of polypoidal lesions were 3.6 in average (range 1-28), and mean largest polyp size was
Table 1 Baseline characteristics of patients with polypoidal choroidal vasculopathy who were treated with intravitreal aflibercept on a fixeddosing schedule

\begin{tabular}{ll}
\hline$N$ (eyes) & 40 \\
Age (years) & $67.0(44-84)$ \\
Sex (male/female) & $27 / 13$ \\
Laterality (right/left) & $15 / 25$ \\
Visual acuity & \\
$\quad$ Mean (ETDRS letters) & $55.1 \pm 18.2$ \\
$\quad$ Median (Snellen) & $20 / 60$ \\
Central subfield macular thickness $(\mu \mathrm{m})$ & $365.2 \pm 104.6$ \\
Location of vascular lesion (eyes) & \\
$\quad$ Subfoveal & $19(47.5 \%)$ \\
$\quad$ Juxtafoveal & $5(12.5 \%)$ \\
$\quad$ Extrafoveal & $16(40.0 \%)$ \\
Area of branching vascular network (mm $\left.{ }^{2}\right)$ & $3.39 \pm 4.10$ \\
Number of polypoidal lesions & $3.6(1-28)$ \\
Size of the largest polypoidal lesion $(\mu \mathrm{m})$ & $286.0 \pm 115.7$ \\
Location of the polyp closest to the foveal center (eyes) & \\
$\quad$ Subfoveal & $1(2.5 \%)$ \\
$\quad$ Juxtafoveal & $12(30.0 \%)$ \\
$\quad$ Extrafoveal & $27(67.5 \%)$ \\
\hline
\end{tabular}

ETDRS Early Treatment of Diabetic Retinopathy Study

$286.0 \pm 115.7 \mu \mathrm{m}$ (range: $131-666 \mu \mathrm{m}$ ). The polyp closest to the foveal center was subfoveal in one eye, juxtafoveral in 12 eyes and extrafoveal in 27 eyes. One eye underwent one session of PDT at 8 months (during maintenance dosing) to treat a marked increase in subretinal fluid and severe vision loss.

\section{Visual acuity}

Mean BCVA improved from 20/80 (55.1 \pm 18.2 letters) at baseline to $20 / 53(64.2 \pm 16.1$ letters $)$ at 12 months $(9.0 \pm$ 18.1 letter gain, $P<.001)$. Significant improvements were observed as early as 1 month after the first injection (Fig. 1a). A total of $35(87.5 \%)$ of 40 eyes did not lose more than 15 letters of vision at 12 months. At this time point, 13 eyes $(32.5 \%)$ had an improvement in BCVA of 15 letters or more, 22 eyes $(55.0 \%)$ had change of $<15$ letters, and five eyes $(12.5 \%)$ lost 15 or more letters (Fig. 2a).

The number of eyes with a visual acuity of 20/40 or better increased from seven eyes $(17.5 \%)$ at baseline to 21 eyes $(52.5 \%)$ at 12 months $(P=.001)$. Additionally, the number of eyes with a visual acuity worse than $20 / 200$ changed from six eyes $(15.0 \%)$ at baseline to two eyes $(5.0 \%)$ at 12 months $(P=.263$; Fig. 2b). Better baseline visual acuity was associated with less visual gain $(r=-.561, P<.001)$ and better BCVA ( $r=.461, P=.003)$ at 12 months. Juxtafoveal location of polyp was the baseline factor related to more visual gain $(P=.044)$. There was no association between the final BCVA and the other baseline factors; polyp size $(P=.437)$, 


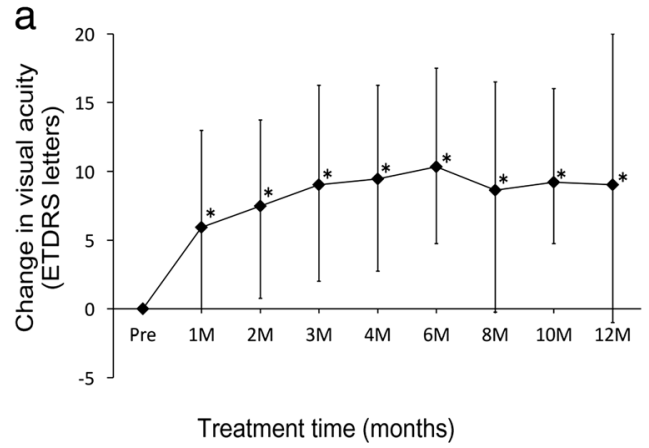

Fig. 1 a Mean change in best-corrected visual acuity and $\mathbf{b}$ central subfield macular thickness in eyes with polypoidal choroidal vasculopathy treated with aflibercept on a fixed dosing schedule (three

polyp number $(P=.339)$, location of polyp $(P=.766)$, location of lesion $(P=.482)$, or BVN area $(P=.885)$.

\section{Anatomical outcomes}

Mean central subfield macular thickness, as measured by OCT, significantly decreased from $365.2 \pm 104.6 \mu \mathrm{m}$ at baseline to $226.8 \pm 50.9 \mu \mathrm{m}$ at 3 months $(P<0.001)$ and to 253.6 $\pm 99.7 \mu \mathrm{m}$ at 12 months $(P<.001$; Fig. $1 \mathrm{~b})$. Qualitative assessments on intraretinal/subretinal/subRPE fluid was performed in 42 eyes, including two eyes that dropped out of the study after 6 months (Fig. 3a). All eyes had a reduction in fluid after the initial loading dose of intravitreal aflibercept (three monthly injections). The macula was completely dry in $32(76.2 \%), 27(64.3 \%)$, and 24 eyes $(60.0 \%)$ at 3, 6, and 12 months respectively.

After switching to the bimonthly injection maintenance schedule, fluid reappeared or increased in $14(33.3 \%)$ eyes at 6 months (Figs. 3a and 4). The group of eyes had a significantly worse final outcome at 12 months than the group of eyes that did not have fluid recurrence. The recurrent group had a lower rate of complete dryness $(23.1 \%$ vs $77.8 \%$, $P<.001)$, a worse BCVA $(55.5 \pm 13.9$ letters vs $68.3 \pm 15.5$ letters, $P=.018$, Fig. 5), and a lower vision gain $(-1.5 \pm 12.4$ vs $+14.1 \pm 18.5$ letters, $P=.005)$. Baseline characteristics of

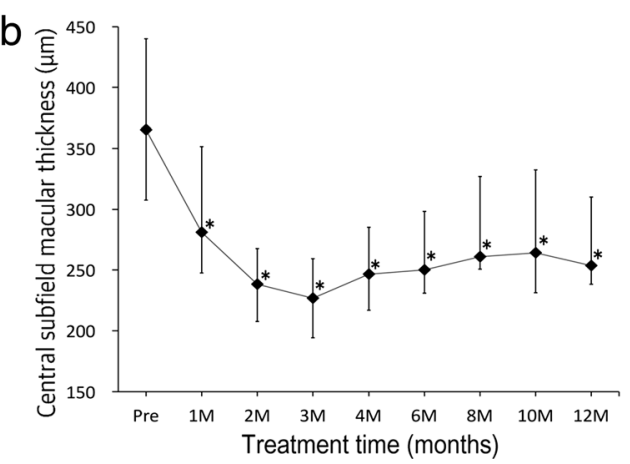

initial monthly injections followed by bimonthly maintenance injections). * indicates a statistically significant difference from baseline $(P<.05)$. Error bars indicate quartile values

these two groups were not significantly different (Table 2). Additionally, six (22.2\%) of 27 eyes that had a dry macula at 6 months had fluid recurrence at 12 months.

A polypoidal lesion was identified on ICGA at baseline in all eyes. After the initial aflibercept loading dose (three monthly injections), complete polyp regression was observed in 26 eyes $(61.9 \%)$, partial regression was observed in ten eyes $(23.8 \%)$, no change was observed in four eyes $(9.5 \%)$, and a new polypoidal lesion was observed in two eyes (4.8\%) (Fig. 3b). Average size of the largest polypoidal lesion at baseline was not different between eyes with and without complete polyp regression at either $3(P=.847)$ or $12(P=.965)$ months. At 12 months, 26 eyes $(66.7 \%)$ had complete polyp regression, and nine eyes $(23.1 \%)$ had developed a new polyp. The BVN area increased in three eyes $(7.1 \%)$ during the loading period (2.65 to $3.32 \mathrm{~mm}^{2}$ ), and in 14 eyes (35.9\%) thereafter ( 2.53 to $5.27 \mathrm{~mm}^{2}$ ), which included four eyes that remained completely dry during the maintenance period (Fig. 6).

\section{Adverse events}

Adverse events were assessed in all of the enrolled patients. Serious adverse events were reported in one patient $(2.1 \%)$, who died of pneumonia. The 73-year-old man had
Fig. 2 a Proportion of patients with improved, maintained, or decreased visual acuity. $\mathbf{b}$ Distribution of visual acuity. $M=$ months
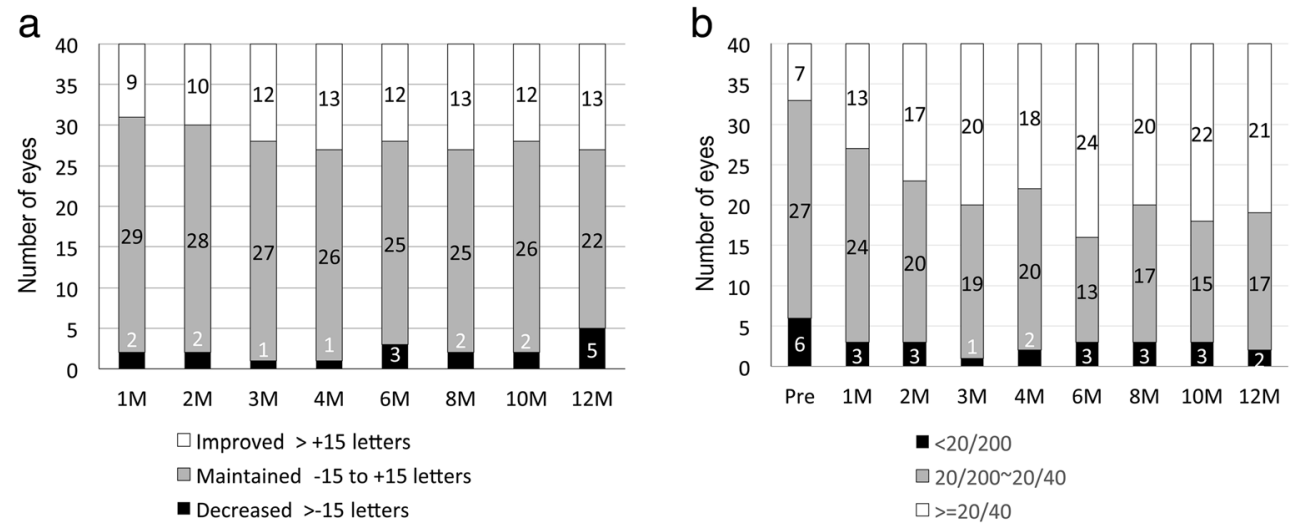
a

Changes in subretinal fluid

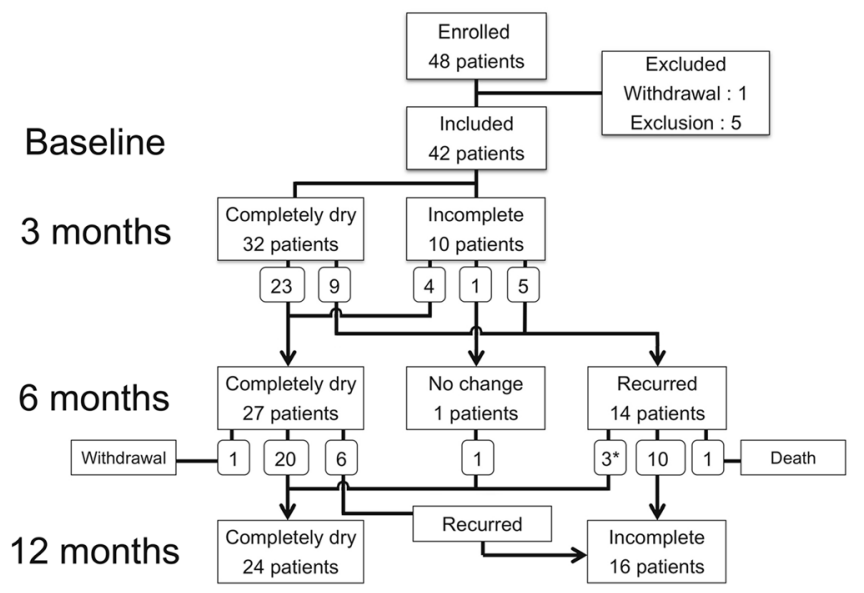

b Changes in polyps

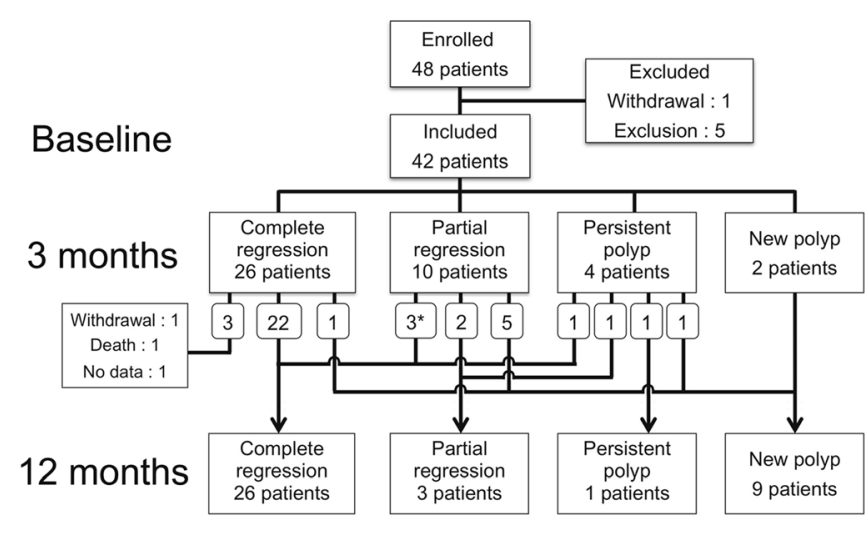

Fig. 3 Flowcharts of a retinal fluid changes assessed with optical coherence tomography, and $\mathbf{b}$ polyp regression assessed with indocyanine green angiography during the study period. * indicates that one eye underwent rescue photodynamic therapy

experienced mild cerebral ischemia 4 months before the event, and administration of aflibercept had been discontinued. Conjunctival hemorrhage related with the injection procedure was the most common adverse event noted. Increase of subretinal hemorrhage was noted in three eyes $(6.3 \%)$. An RPE tear also occurred in two eyes $(4.2 \%)$. Other adverse events included urticaria of the skin (one subject), tinnitus (one subject), and headache (one subject).

\section{Discussion}

Our prospective study of a fixed-dose aflibercept regimen for treating eyes with treatment-naïve PCV had favorable visual outcomes, with BCVA maintained or improved in $87.5 \%$ (35 of 40 eyes). On average, BCVA improved by 9 letters over 12 months, and approximately half of all patients had a BCVA of 20/40 or better. However, there was a group of patients that responded poorly to maintenance dosing schedule (injection every 2 months).

The first line of treatment for PCV is somewhat debatable, unlike for typical AMD. Several studies have compared the efficacies of ranibizumab and PDT, but have reported conflicting results in anatomical and functional outcomes. The EVEREST study [8] was a prospective, multi-center clinical trial that compared the efficacy of PDT combined with intravitreal ranibizumab, verteporfin PDT monotherapy, and intravitreal ranibizumab monotherapy for treating eyes with PCV. Complete regression of polypoidal lesions occurred more frequently in eyes receiving PDT, and there was no difference in visual acuity between the combination and ranibizumab monotherapy groups. Kokame et al. [6] reported polyp regression in $33 \%$ of eyes 6 months after beginning monthly ranibizumab injections. Based on these results, Koh et al. [18] suggested that verteporfin PDT, with or without ranibizumab, should be the first-line treatment in eyes with $\mathrm{PCV}$ to induce polyp regression.

However, verteporfin PDT monotherapy has been shown to result in smaller visual gains than intravitreal ranibizumab monotherapy, even though PDT results in a more effective reduction in central macular thickness and a more frequent regression of polyps $[8,11,12]$. Performing PDT resolved intra- and/or subretinal fluid in eyes with PCV that did not respond to anti-VEGF treatments, but visual acuity was not maintained [19]. Potential side-effects of PDT, including damage to photoreceptors and the RPE, were thought to be the cause of this anatomical and functional outcome discrepancy. Therefore, it was suggested that polyp regression and exudative improvements do not necessarily translate into a good visual outcome [12]. Nevertheless, anatomical outcomes are important for securing long-term results because persistent subretinal fluid and/or a sudden, massive submacular hemorrhage can result in permanent visual impairment.

In the present study, a standard aflibercept loading dose (three monthly injections) was initially administered and was followed by injections administered every 2 months. This treatment schedule was the same as that used in the VIEW studies [13]. Complete regression of polypoidal lesions occurred in $61.9 \%$ of patients at 3 months and in $66.7 \%$ of patients at 12 months. This regression rate was comparable to that obtained with PDT and other studies examining aflibercept therapy (55.4-75.0\% polyp closure rate) [14-17]. It hasd been shown that aflibercept has a stronger affinity for VEGF-A than ranibizumab, and also that it blocks VEGF-B and PIGF [20,21]. These drug biological characteristics would explain the higher polyp closure rate with aflibercept than with ranibizumab.

The VIEW 1 and VIEW 2 trials [13] demonstrated a mean gain of 7.9 and 8.9 letters respectively in patients with wet AMD. Another study also showed a $0.14 \log$ MAR unit 


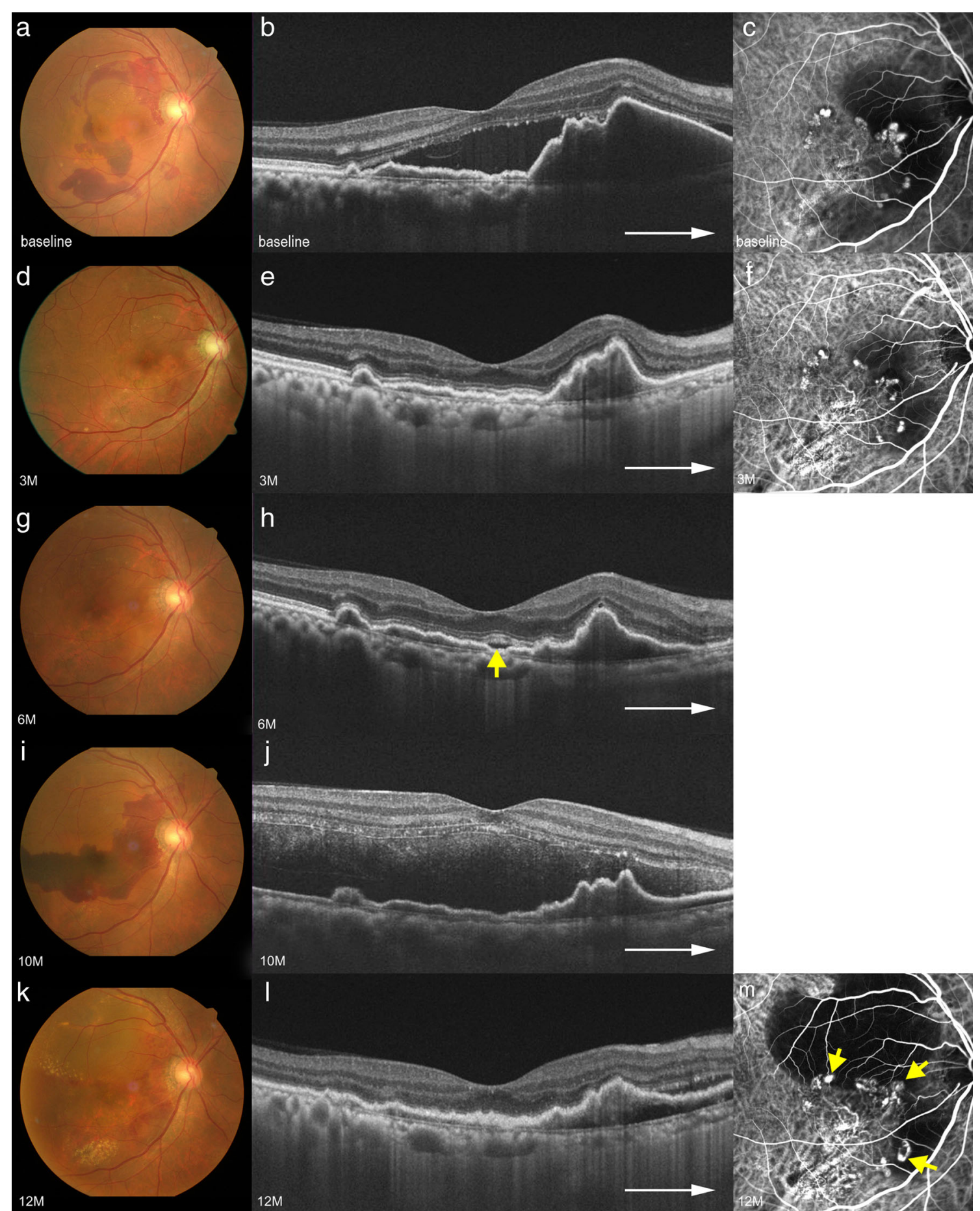

Fig. 4 Fundus photographs (left column), optical coherence tomography images (center column), and indocyanine green angiography (ICGA, right column) images of a 60 -year-old man with polypoidal choroidal vasculopathy $(\mathrm{PCV})$ treated with a fixed-dosing regimen of aflibercept (three initial monthly injections followed by bimonthly injections). a-c At baseline, subretinal fluid (SRF) and a pigment epithelial detachment (PED) related to PCV are apparent. Visual acuity was 20/40. A branching

(equivalent to 7 ETDRS letters) gain in visual acuity in eyes with PCV treated with aflibercept [17]. In these studies and ours, visual acuity was maintained between 3 and 12 months vascular network and polypoidal vessels are visible on ICGA. $\mathbf{d}-\mathbf{f}$ At 3 months, SRF had resolved even though polypoidal lesions had only partially regressed. $\mathbf{g}, \mathbf{h}$ New SRF is visible 6 (arrow). I, j A subretinal hemorrhage and a large PED are noted. $\mathbf{k}-\mathbf{m}$ Although SRF had resolved, visual acuity had decreased to 20/125 and new polypoidal lesions developed, as visible on ICGA (arrows)

while subjects received injections every 2 months. Nonetheless, in the current study, the proportion of eyes that lost $<15$ letters changed from $98 \%$ at 3 months to $88 \%$ at 
Fig. 5 Mean visual acuity in eyes with polypoidal choroidal vasculopathy treated with aflibercept every 2 months following three monthly loading injections. An increase in subretinal/intraretinal fluid was noted in 14 eyes after extending the treatment interval from 1 to 2 months. Patients with recurrent fluid had a significantly worse visual acuity than patients with no recurrence. * indicates a statistically significant difference between groups $(P<.05)$. Error bars indicate quartile values

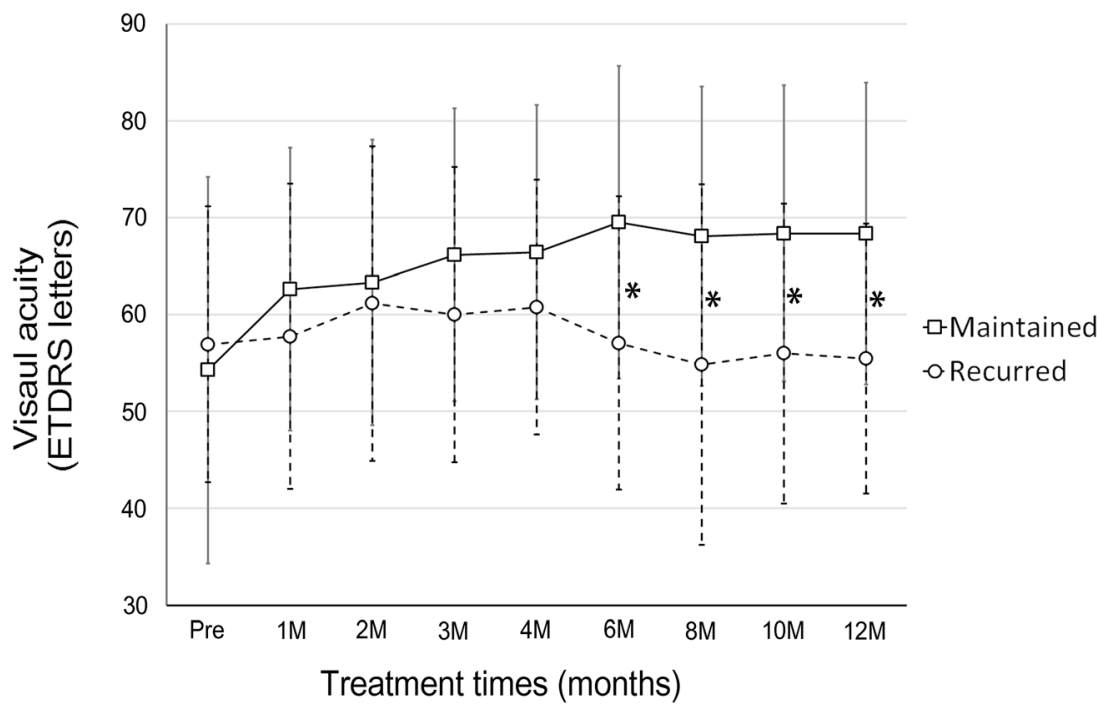

Table 2 Characteristics of patients with polypoidal choroidal vasculopathy who had a maintained or recurred sub/ intraretinal fluid after extending the aflibercept treatment interval from 1 month to 2 months

\begin{tabular}{|c|c|c|c|}
\hline & Maintained & Recurred & $P$ value \\
\hline$N$ (eyes) & $27(67.5 \%)$ & $13(32.5 \%)$ & \\
\hline Sex (male/female) & $18 / 9$ & $9 / 4$ & $>.999^{\mathrm{a}}$ \\
\hline Age (years) & $67.7 \pm 9.6$ & $65.6 \pm 8.4$ & $.508^{\mathrm{b}}$ \\
\hline Laterality (right/left) & $9 / 18$ & $6 / 7$ & $.498^{\mathrm{a}}$ \\
\hline Location of vascular lesion (eyes) & & & $>.999^{\mathrm{a}}$ \\
\hline Subfoveal & 13 & 6 & \\
\hline Juxtafoveal & 3 & 2 & \\
\hline Extrafoveal & 11 & 5 & \\
\hline Area of branching vascular network $\left(\mathrm{mm}^{2}\right)$ & $3.86 \pm 4.88$ & $2.41 \pm 1.24$ & $.732^{\mathrm{b}}$ \\
\hline Number of polyps & $3.3(1-11)$ & $3.0(1-28)$ & $.632^{\mathrm{b}}$ \\
\hline Size of the largest polypoidal lesion $(\mu \mathrm{m})$ & $288.5 \pm 101.4$ & $280.7 \pm 145.5$ & $.441^{\mathrm{b}}$ \\
\hline Location of the polyp closest to the fovea & & & $>.325^{\mathrm{a}}$ \\
\hline Subfoveal & 0 & 1 & \\
\hline Juxtafoveal & 9 & 3 & \\
\hline Extrafoveal & 18 & 9 & \\
\hline Central subfield macular thickness $(\mu \mathrm{m})$ & $368.4 \pm 104.6$ & $358.5 \pm 108.7$ & $.842^{\mathrm{b}}$ \\
\hline \multicolumn{4}{|l|}{ Completely dry (eyes) } \\
\hline Month 3 & $19(70.4 \%)$ & $8(61.5 \%)$ & $.721^{\mathrm{a}}$ \\
\hline Month 6 & $26(96.3 \%)$ & $0(0 \%)$ & $<.001^{\mathrm{a}}$ \\
\hline Month 12 & $21(77.8 \%)$ & $3(23.1 \%)^{\mathrm{c}}$ & $<.001^{\mathrm{a}}$ \\
\hline \multicolumn{4}{|l|}{ Visual acuity (letters) } \\
\hline Baseline & $54.3 \pm 20.0$ & $56.9 \pm 14.2$ & $.670^{\mathrm{b}}$ \\
\hline Month 3 & $66.1 \pm 15.1$ & $60.0 \pm 15.2$ & $.276^{\mathrm{b}}$ \\
\hline Month 6 & $69.5 \pm 16.1$ & $57.1 \pm 15.1$ & $.019^{\mathrm{b}}$ \\
\hline Month 12 & $68.3 \pm 15.5$ & $55.5 \pm 13.9$ & $.018^{\mathrm{b}}$ \\
\hline \multicolumn{4}{|l|}{ Visual acuity gain (letters) } \\
\hline Month 3 & $11.9 \pm 14.5$ & $3.1 \pm 7.3$ & $.039^{\mathrm{b}}$ \\
\hline Month 6 & $15.3 \pm 15.5$ & $0.2 \pm 14.9$ & $.005^{\mathrm{b}}$ \\
\hline Month 12 & $14.1 \pm 18.5$ & $-1.5 \pm 12.4$ & $.005^{\mathrm{b}}$ \\
\hline
\end{tabular}

${ }^{a}$ indicates $P$ value calculated with a Fisher's exact test

b indicates $P$ value calculated with a Mann-Whitney $U$ test

${ }^{\mathrm{c}}$ includes one eye that underwent rescue photodynamic therapy 


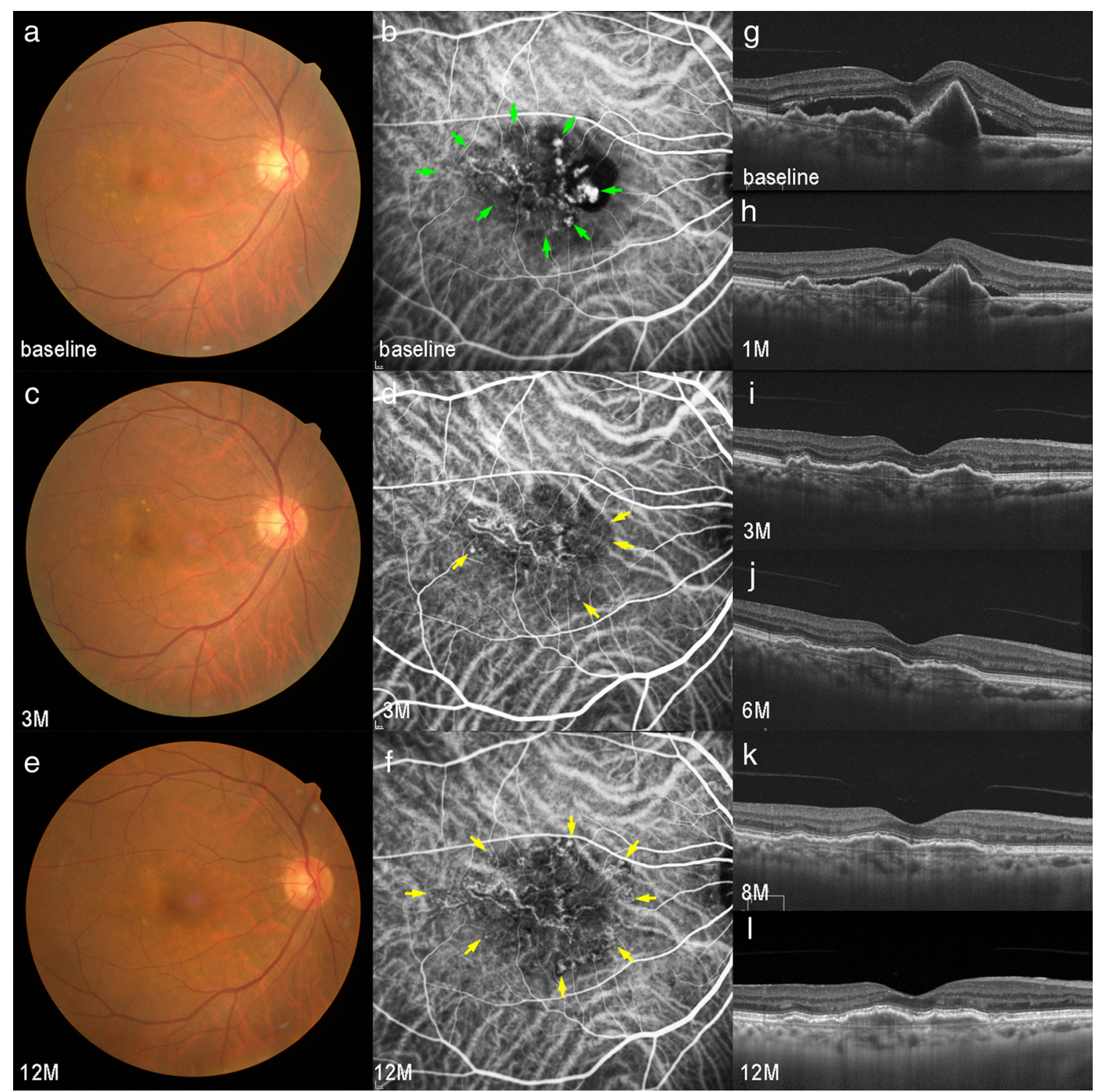

Fig. 6 Fundus photographs (left column), indocyanine green angiography images (ICGA, middle column), and optical coherence tomography (OCT) images (right column) obtained from a 63-year-old man with polypoidal choroidal vasculopathy $(\mathrm{PCV})$ treated with fixeddosing aflibercept. a, b At baseline, exudative changes related to PCV are apparent with branching vascular network (BVN) and polypoidal vessels

12 months. This may have occurred because of retinal fluid recurrence in approximately one-third of eyes after the treatment interval was extended to 2 months (Fig. 4). These findings are probably related to the 'saw teeth' phenomenon in central macular thickness that has been observed previous studies [13, 17]. However, some of our patients developed a subretinal hemorrhage (Fig. 5) or a substantial increase in subretinal fluid and required rescue PDT. Not surprisingly, eyes with increased fluid at 6 months also had a worse final visual acuity. Therefore, the conventional bimonthly maintenance dosing schedule is suboptimal for some patients, and more intensive treatments (e.g., more frequent injections and/ noted on ICGA images (arrows). c, d Although polyps present at baseline completely regressed after three monthly injections, BVN growth and new polyp formation occurred at 3 months (arrows). e, f New polyps and BVN growth were still present at 12 months (arrows on ICGA image). g-I Study OCT images shown that the macula remained dry from 3 to 12 months

or PDT) may be needed. Unfortunately, no associations between fluid recurrence and baseline characteristics were found, and we cannot predict which patients will need more intensive treatments. However, vision gain after loading injections and fluid status 6 months into treatment may be predictors of long-term outcomes.

New polyp formation and branching BVN enlargement were observed on ICGA during continued injections at fixed 2 month intervals. Another study reported similar findings, showing that the proportion of patients with a smaller lesion size than at baseline decreased from $25.6 \%$ at 3 months to $13.4 \%$ at 12 months [17]. It is worth mentioning that 4 eyes 
included in the current study had a BVN size increase even though the macula remained dry throughout the maintenancedosing period. It may be that threshold anti-VEGF agent levels needed to prevent PCV growth are different than those needed to suppress exudative changes. It may also be that mechanisms not related to VEGF are involved in BVN growth. The relationship between PCV lesion growth and long-term outcomes should be studied in eyes without exudative changes to better understand these other mechanisms.

There was one serious adverse event of death, of which cause was pneumonia. Although it did not appear to be related to intravitreal aflibercept that had been discontinued 5 months before.

Our study had several limitations. We did not have a comparison control group. The examined number of cases was relatively small and the follow-up period was relatively short. However, the fact that this was a prospective study on a homogeneous group of PCV patients that included data from different sites makes our data robust.

In conclusion, 12 month anatomical and functional outcomes were favorable after administering aflibercept on a fixed dosing schedule ( 3 monthly injections followed by bimonthly injections) to treat treatment-naïve PCV. However, fluid recurrence was observed in one-third of patients after extending the treatment interval to 2 months. Because final outcomes in these patients were poor, a more flexible treatment strategy should be used. Larger studies with a longer follow-up period that compare treatment strategies (e.g., prore-nata or treat-and-extend dosing) are warranted.

Acknowledgment Supported by Bayer Korea (Seoul, Republic of Korea)

\section{Compliance with ethical standards}

Funding Bayer Korea (Seoul, Republic of Korea) provided funding and investigational drugs for this study.

Conflict of interest Joo Eun Lee: consultant for Allergan, Bayer, Novartis, honorarium from Allergan, Bayer, Novartis, Alcon, research grant from Allergan, Bayer. Hyun Woong Kim: honorarium and travel grant from Bayer, Novartis. Woohyok Chang: Consultant for Bayer, Novartis, honorarium from Bayer, research grant from Novartis. Ji Eun Lee: Consultant for Allergan, Bayer, Novartis, honorarium from Allergan, Bayer, Novartis, Bausch \& Lomb, research grant from Bayer. The following authors have no financial disclosures: Jae Pil Shin, Yu Cheol Kim, Sang Joon Lee, In Young Chung, Kwang Soo Kim, Sung Who Park.

Ethical approval All procedure performed in studies involving human participants were in accordance with the ethical standards of the institutional and national research committee and with the 1964 Helsinki Declaration and its later amendments or comparable ethical standards.

Informed consent Informed consent was obtained from all individual participants included in the study.

\section{References}

1. Lafaut BA, Leys AM, Snyers B, Rasquin F, De Laey JJ (2000) Polypoidal choroidal vasculopathy in Caucasians. Graefes Arch Clin Exp Ophthalmol 238:752-759

2. Maruko I, Iida T, Saito M, Nagayama D, Saito K (2007) Clinical characteristics of exudative age-related macular degeneration in Japanese patients. Am J Ophthalmol 144:15-22

3. Rosenfeld PJ, Brown DM, Heier JS et al (2006) Ranibizumab for neovascular age-related macular degeneration. N Engl J Med 355: 1419-1431

4. Brown DM, Kaiser PK, Michels M et al (2006) Ranibizumab versus verteporfin for neovascular age-related macular degeneration. $\mathrm{N}$ Engl J Med 355:1432-1444

5. Gomi F, Sawa M, Sakaguchi H, Sasamoto Y, Suzuki M, Tzujikawa M (2008) Efficacy of intravitreal bevacizumab for polypoidal choroidal vasculopathy. Br J Ophthalmol 92:70-73

6. Kokame GT, Yeung L, Lai JC (2010) Continuous anti-VEGF treatment with ranibizumab for polypoidal choroidal vasculopathy: 6month results. Br J Ophthalmol 94:297-301

7. Tsujikawa A, Ooto S, Yamashiro K, Tamura H, Otani A, Yoshimura N (2010) Treatment of polypoidal choroidal vasculopathy by intravitreal injection of bevacizumab. Jpn J Ophthalmol 54:310-319

8. Koh A, Lee WK, Chen LJ et al (2012) EVEREST study: efficacy and safety of verteporfin photodynamic therapy in combination with ranibizumab or alone versus ranibizumab monotherapy in patients with symptomatic macular polypoidal choroidal vasculopathy. Retina 32:1453-1464

9. Sayanagi K, Gomi F, Sawa M, Ohji M, Tano Y (2007) Long-term follow-up of polypoidal choroidal vasculopathy after photodynamic therapy with verteporfin. Graefes Arch Clin Exp Ophthalmol 245:1569-1571

10. Tsuchiya D, Yamamoto T, Kawasaki R, Yamashita H (2009) Twoyear visual outcomes after photodynamic therapy in age-related macular degeneration patients with or without polypoidal choroidal vasculopathy lesions. Retina 29:960-965

11. Inoue M, Arakawa A, Yamane S, Kadonosono K (2013) Long-term outcome of intravitreal ranibizumab treatment, compared with photodynamic therapy, in patients with polypoidal choroidal vasculopathy. Eye (Lond) 27:1013-1020

12. Oishi A, Kojima H, Mandai M et al (2013) Comparison of the effect of ranibizumab and verteporfin for polypoidal choroidal vasculopathy: 12-month LAPTOP study results. Am J Ophthalmol 156:644651

13. Heier JS, Brown DM, Chong V et al (2012) Intravitreal aflibercept (VEGF trap-eye) in wet age-related macular degeneration. Ophthalmology 119:2537-2548

14. Hosokawa M, Shiraga F, Yamashita A et al (2015) Six-month results of intravitreal aflibercept injections for patients with polypoidal choroidal vasculopathy. Br J Ophthalmol 99:1087-1091

15. Inoue M, Arakawa A, Yamane S, Kadonosono K (2014) Short-term efficacy of intravitreal aflibercept in treatment-naïve patients with polypoidal choroidal vasculopathy. Retina 34:2178-2184

16. Oishi A, Tsujikawa A, Yamashiro K et al (2015) One-year result of aflibercept treatment on age-related macular degeneration and predictive factors for visual outcome. Am J Ophthalmol 159:853-860

17. Yamamoto A, Okada AA, Kano M et al (2015) One-year results of intravitreal aflibercept for polypoidal choroidal vasculopathy. Ophthalmology 122:1866-1872

18. Koh AH, Chen LJ, Chen SJ et al (2013) Polypoidal choroidal vasculopathy: evidence-based guidelines for clinical diagnosis and treatment. Retina 33:686-716

19. Byon IS, Kwon HJ, Kim SI, Shin MK, Park SW, Lee JE (2014) Reduced-fluence photodynamic therapy in polypoidal choroidal 
vasculopathy nonresponsive to ranibizumab. Ophthalmic Surg Lasers Imaging Retina 45:534-541

20. Holash J, Davis S, Papadopoulos N et al (2002) VEGF-Trap: a VEGF blocker with potent antitumor effects. Proc Natl Acad Sci U S A 99:11393-11398
21. Papadopoulos N, Martin J, Ruan Q et al (2012) Binding and neutralization of vascular endothelial growth factor (VEGF) and related ligands by VEGF Trap, ranibizumab and bevacizumab. Angiogenesis 15:171-185 